\title{
Introduction to the special issue: Employment and vocational rehabilitation considerations for people with disabilities during and after the COVID-19 pandemic
}

\author{
Phillip D. Rumrill ${ }^{\mathrm{a}, *}$, Lynn C. Koch ${ }^{\mathrm{b}}$ and David R. Strauser ${ }^{\mathrm{c}}$ \\ ${ }^{a}$ University of Kentucky, Lexington, KY, USA \\ ${ }^{\mathrm{b}}$ University of Arkansas, Fayetteville, AR, USA \\ ${ }^{\mathrm{c}}$ University of Illinois, Urbana-Champaign, Champaign, IL, USA
}

In March of 2020, the World Health Organization declared a worldwide pandemic in response to the Coronavirus-2019 (COVID-19) outbreak. As of this writing, there have been more than 16 million confirmed cases of the virus in the United States and 72 million worldwide. Over 300,000 Americans who have acquired COVID-19 have died, making it the current leading cause of death in the United States. People with some types of disabilities, especially those with multiple disadvantaging characteristics and intersectionalities based on race/ethnicity, age, socioeconomic status, and gender, are both more likely to contract the virus and more likely to experience severe or fatal symptoms. Phase $1 \mathrm{~A}$ of the effort to vaccinate Americans began in December 2020, prioritizing health care workers and long-term care residents as the first people to be inoculated against the virus. However, experts warn that it could take until mid-2021 until all of those who desire and are medically authorized for the vaccine receive it, and we could continue to witness unprecedented

*Address for correspondence: Phillip D. Rumrill, University of Kentucky, Human Development Institute, 126 Graham Avenue, Lexington, Kentucky 40508, USA. Tel.: +1 330524 1598; E-mail: phillip.rumrill@uky.edu. increases in confirmed cases and deaths in the months to come, especially if we do not take universal precautions to protect ourselves and others.

Although millions of Americans have recovered from COVID-19, they often experience lingering symptoms. The Centers for Disease Control and Prevention is investigating the long-term health effects of COVID-19 and will continue to follow individuals with lingering symptoms for years to come to determine whether these symptoms eventually resolve or if they result in permanent disabilities. Among the most commonly reported lasting symptoms are fatigue, shortness of breath, cough, joint pain, and chest pain. Other long-term symptoms include "brain fog," depression, muscle pain, headache, intermittent fever, and heart palpitations. Less frequently reported but still intrusive symptoms include inflammation of the heart muscle, lung functioning abnormalities, acute kidney injury, hair loss, rash, loss of smell and taste, sleep problems, and concentration and memory problems.

Not to be underestimated are the mental health effects of the pandemic. The stress of life during these unprecedented times can manifest in fear and anxiety about one's own health, the health of loved ones, finances, job security, and reduced access to 
formal and natural supports; changes in sleeping patterns; changes in eating habits; difficulty concentrating; worsening of pre-existing conditions (both physical and psychiatric); and increased use of alcohol, tobacco, and other substances. If individuals have lost loved ones to COVID-19 and were unable to be at their hospital bedsides for end-of-life visits, these mental health effects can be complicated by profound grief.

Because many people with disabilities are at increased risk of experiencing severe symptoms of COVID-19, self-isolation to protect themselves from contracting the virus can lead to loneliness and despair. Individuals who live in congregate settings (e.g., nursing homes) have also been identified as being at increased risk for mental health issues. Also putting individuals at risk of mental health issues during COVID-19 are pre-existing mental health conditions that are exacerbated by the stressors of the pandemic, the greater likelihood that people with disabilities live in poverty and experience higher rates of homelessness than the general population, and the increased likelihood of being from marginalized populations without appropriate access to healthcare. Also, of importance to note is that fears about contracting the virus may lead individuals to cancel appointments with medical and healthcare providers, thus putting themselves at risk of sub-optimal management and potential worsening of their disabling conditions.

There is no doubt that people with disabilities are especially likely to be negatively affected by pandemic-related changes in the manners in which people worldwide work, transact business, communicate with one another, socialize, protect themselves and their families, learn, access healthcare, and participate in political processes. In the new COVIDdriven American and global workplaces, people with disabilities have been disproportionally removed from the labor force, often due to difficulties in arranging technology to enable them to work at home or to the lack of available in-person on-the-job supports.

Among rehabilitation professionals, healthcare providers, and disability advocates, it is well established that people with disabilities require significant supports to navigate the risks and complexities of the COVID-19 pandemic. Rehabilitation professionals and other service providers have found it difficult to pivot to entirely virtual services, and telehealth platforms for medical services have not entirely proven themselves as a substitute for in-person treatment, especially for people with complex disability-related needs. Consequently, vocational rehabilitation (VR) professionals are challenged with how to provide services to people with disabilities during a time when, collectively, they are likely to need our support more than ever before. We must find ways for continuing to connect with consumers and reduce their sense of isolation and hopelessness.

We must also ensure that people with disabilities benefit maximally from the post-COVID economic recovery that most experts predict will be spurred by the worldwide distribution of vaccines. To actualize this imperative, it is essential for VR professionals to understand the severe and profound impact that COVID-19 has had on the disability community; the ways in which the global workforce has changed; the use of assistive and communication technology to support telecommuting and home-based employment; how and why certain industries have flourished during the pandemic while others have been decimated; which sectors of the economy and job markets are likely to have the most fertile opportunities for workers with disabilities in the post-COVID era; and how the traumatic experience of this global pandemic is likely to affect commerce, public health, education, and employment for many years to come.

Accordingly, the purpose of this special issue is to highlight research and scholarship in the interdisciplinary field of VR that address important implications of the COVID-19 pandemic and its aftermath for the disability community and for rehabilitation professionals. Some of the articles contributed by our colleagues are directly targeted to the COVID outbreak, whereas others focus on topics and research findings that pose unique implications for VR services during and after COVID-19. Sheppard-Jones \& Avellone, et al. (2021) opened this special issue and described COVID-19-related issues facing people with developmental disabilities. Schall et al. (2021) examined employment files to see the unique effects that the pandemic has had on individuals with intellectual and developmental disabilities. Umucu (2021) presented a COVID-era survey in which people with disabilities and other health conditions reported significant levels of worry about their health and job loss as a result of the pandemic. Rumrill et al. (2021) investigated differences in the employment experiences and concerns of Latinx Americans and Caucasian Americans with multiple sclerosis, and they applied those findings to the current global public health crisis. Adams et al. (2021) reported qualitative results from a focus 
group study of isolation and social distancing among working-age adults with spinal cord injuries. Strauser et al. (2021) demonstrated the validity and clinical utility of a scale to measure work adjustment among people with disabilities during COVID-19. Kaya et al. (2021) examined differential service patterns and employment outcomes among transition-age youth with learning disabilities who received services from the state-Federal VR program, including a discussion of the COVID implications of their findings. Sheppard-Jones \& Goldstein et al. (2021) examined the impact of the pandemic on the employment, health, and community participation of Americans with disabilities through the lens of universal design.
It is our hope that this issue presents content that will aid JVR readers in providing responsive VR services to people with a wide range of disabling conditions as the global community continues to struggle with this, the most far-reaching and costly (in every sense of the word) public health crisis in the history of the world. We express our sincere gratitude to the above-mentioned authors who contributed such excellent works to this issue. We wish to convey special thanks to Dr. Paul Wehman, Editor of JVR, and Ms. Hannah Seward, JVR's Production Manager, for the opportunity to undertake and complete this project. 